\title{
Comportamiento de dos escalas predictoras de infección de herida operatoria en cirugía de bypass coronario: NNIS y Toronto en población chilena
}

\author{
PATRICIA LÓPEZ F. y ALONSO CORREA L.
}

\author{
Behavior of two index scale risk of surgical wound infection in coronary \\ artery bypass graft: NNIS and Toronto score, in Chilean patients
}

A multicenter case-control pilot study was conducted (71/213) to know the predictive value of two risk scales for nosocomial wound infections, NNIS and Toronto score, applied to coronary artery bypass graft surgery in six hospitals in Santiago- Chile, from 1998 to 2002. A straight correlation of wound infection risk and Intensive Care Unit stay beyond three days, sub-group of the Toronto scale, was identified. Toronto scale, according to its four groups of clinical risk, is therefore an effective tool to adjust rates and make comparisons between hospitals that perform this type of surgery.

Key words: Coronary artery bypass grafts, Risk index, Nosocomial infection.

Palabras claves: Injerto de bypass coronario; Índice de riesgo; Infección nosocomial.

\section{Introducción}

En la infección nosocomial de sitio quirúrgico (ISQ), participan múltiples factores como son los propios del paciente y los del entorno asistencial. Dependiendo del tipo de cirugía y del grado de compromiso secundario a la infección, esta complicación consume gran cantidad de recursos, aumentando la estadía hospitalaria y originando otros costos directos e indirectos de índole variable, difíciles de dimensionar ${ }^{1}$.

En Chile, según informes de vigilancia nacional elaborados por el Ministerio de Salud², las ISQ han disminuido progresivamente su incidencia evidenciando un mayor impacto en cirugía de hernia inguinal y colecistectomía por laparoscopia. Esta tendencia también se ha observado en cirugía de bypass coronario, reportándose en el año 2003 una tasa de 3,7 por 100 eventos.

La ejecución de bypass coronario corresponde a una cirugía altamente invasora que involucra a un equipo multidisciplinario y que conlleva gran cantidad de prácticas asistenciales. Estas últimas están íntimamente relacionadas a la adquisición de ISQ, como probablemente también lo sean las diferentes características de la población atendida. Esto determina un particular y diferente comportamiento de las tasas de ISQ en cada centro informante del sistema público ${ }^{3}$. El acotar las diferencias y atribuirlas al tipo de atención asistencial, necesariamente implica un análisis previo por tasas ajustadas. Así entonces, la identificación indirecta, por centro, de la mejor práctica asistencial médico-quirúrgica, contribuiría a una política de centralización de recursos o más simplemente y cercano, favorecería una mejor libre elección de los pacientes con los medios necesarios para optar a ella.

En E.U.A., la Joint Commission on Hospital Acreditation recomienda dos índices: Study of Efficacy of Nosocomial Infection Control (SENIC), y National Nosocomial Infection

Departamento de Infecciones y Epidemiología Hospitalaria, Hospital Militar del General Luis Felipe Brieba Arán, Santiago, Chile (PLF).

Estudiante de Medicina, Facultad de Medicina Universidad de Chile, Santiago, Chile (ACL). 
Surveillance (NNIS), para ajustar y comparar las tasas de ISQ entre diferentes centros de salud hospitalarios ${ }^{4}$. En Chile no existe claro consenso de las ventajas de la estratificación por riesgos en la vigilancia de infecciones intrahospitalarias $(\mathrm{IIH})$, y es probable que un estudio como el desarrollado, que permita identificar una puntuación, eventualmente aporte hacia una estratificación en el ámbito nacional, en al menos algunos parámetros de riesgo ${ }^{5,6}$.

Al respecto se conoce al menos dos escalas que miden riesgo de ISQ: NNIS y Toronto, la primera con una profusa aceptación y aplicación en centros extranjeros, y la segunda con un claro diseño de estudio al momento de su desarrollo ${ }^{7}$. Ambas entonces se seleccionaron para determinar si la clasificación del riesgo de ISQ por NNIS o Toronto, aplicadas a población chilena, son predictoras en pacientes de ambos sexos, con infección de esternotomía por cardiocirugía de bypass, en comparación con los pacientes que no cursaron con la infección ${ }^{8}$.

La escala NNIS, conocida predictora de ISQ desde 1970, y reconocidos reportes de su aplicación ${ }^{9,10}$, es utilizada voluntariamente por cerca de 300 hospitales de al menos 100 camas, en 42 estados de los E.U.A. Fue modificada en 1990, $3^{\circ}$ Decenal de IIH del $\mathrm{CDC}^{11}$ y es sometida a revisión regularmente respecto a su comportamiento en la vigilancia de los eventos nosocomiales. Las variables que contiene son: el índice ASA desarrollado en 1960 y de escasa precisión científica según Dripps y otros investigadores ${ }^{12,13}$, el tiempo quirúrgico y, en tercer lugar, la clasificación de la herida operatoria. No considera variables de complicaciones asociadas al evento.

El índice Toronto se diseñó en Canadá con posterioridad a un estudio de 11.508 pacientes operados de bypass coronario, como un predictor específico para la infección de esternotomía relacionada a esta cirugía. De las variables estudiadas, finalmente se excluyó la variable experiencia del cirujano, relevante en los resultados finales. La escala quedó conformada entonces por complicaciones asociadas a la cirugía (reintervención), una relacionada a la atención asistencial (sobreestadía en UCI), un factor propio del paciente (diabetes mellitus), y una variable respecto a la técnica quirúrgica (elección del tipo de vaso sanguíneo empleado para el bypass).

Ambas escalas, por lo tanto sustentan diferencias fundamentales que no permiten comparaciones entre poblaciones medidas por una $\mathrm{u}$ otra indistintamente.

Para efectos de este estudio en población chilena, se plantean entonces como objetivos:
- Determinar la escala que se comporta como un mejor predictor de ISQ.

- Identificar asociaciones entre ISQ y las subvariables de las escalas.

- Identificar la asociación de los casos con fallecimiento durante la hospitalización.

En suma, se pretende identificar una escala predictora de ISQ en población chilena operada de bypass coronario con visión en la comparación por tasas ajustadas por una de estas escalas, entre diferentes centros asistenciales que efectúan esta cirugía en el país

\section{Pacientes y Método}

Estudio piloto, caso-control, retrospectivo, multicéntrico de 6 hospitales; dos del Sistema Público de Salud: Hospital San Juan de Dios y Hospital Clínico San Borja Arriarán; uno universitario: Hospital Clínico de la Universidad de Chile, y tres de las Fuerzas Armadas y de Orden, ubicados en la Región Metropolitana de Santiago: Hospital Clínico de la Fuerza Aérea de Chile General Raúl Yazigi J, Hospital de la Dirección de Previsión de Carabineros de Chile y Hospital Militar del General Luis Felipe Brieba Arán. Se seleccionó como período de estudio cinco años: 1998 al 2002.

El evento de estudio primario fue el comportamiento de ambas escalas en pacientes operados de bypass coronario, con y sin infección de herida operatoria. Los casos se obtuvieron de los registros de infecciones intrahospitalarias, los controles de los listados quirúrgicos y los datos desde los registros médicos de cada paciente.Se incluyeron pacientes adultos de ambos sexos, beneficiarios del Fondo Nacional de SaludFONASA, Fuerzas Armadas y de Orden e ISAPRES. Se excluyeron pacientes infectados por VIH, pacientes con inmunodeficiencia y sometidos a trasplante de órganos sólidos. Por aplicación de test exacto de Fisher se determinó un tamaño de muestra de 87 casos y 261 controles. En la calificación del riesgo por escalas se utilizaron los siguientes valores aceptados:

- NNIS = 0: riesgo bajo; 1 : riesgo mediano; 2: riesgo mediano-alto; 3: riesgo alto.

- Toronto $=0$ a 5: muy bajo riesgo; 5,5 a 9: riesgo bajo; 9,5 a 11,5: riesgo mediano; mayor a 12: riesgo alto.

La búsqueda de información se centró en los siguientes criterios y variables dicotomizadas: 
- Independientes: duración de la cirugía (> 5 hs); ASA (> 2); clasificación de la herida quirúrgica (limpia, contaminada, sucia, infectada); diabetes mellitus; uso de arteria mamaria interna; reintervención; UCI (> 3 días).

- Dependientes: ISQ; fallecimiento durante la hospitalización.

- Confundentes: edad (<64, > 70 años); sexo; antibioprofilaxis, circulación extra corpórea (CEC).

- De razón: número de puentes (> a 2).

La clasificación en escala Toronto (> 9) y escala NNIS (>2) fue efectuada por los investigadores, en acuerdo con el puntaje asignado a las variables independientes comprendidas en cada índice. Para el análisis estadístico se exigió 95\% de confianza. Se efectuó identificación de asociaciones por $\chi^{2}$ con corrección de Yates y test exacto de Fisher. Además regresión logística para variables agrupada y lineal para edad/ISQ. La sensibilidad y especificidad de las escalas se evaluó por curvas ROC. Para los análisis se recurrió a los programas computacionales Excel y Stata 7.0.

\section{Resultados}

Luego de efectuada la recolección de la información, se consiguió un tamaño de muestra final de 71 casos y 213 controles, 18\% menor al estimado, con pareo cercano al $100 \%$. La participación de pacientes caso-control de cada centro se presenta en la Tabla 1.

Los casos se distribuyeron en $63,4 \%$ varones y $36,6 \%$ mujeres. El promedio de edad en el sexo masculino fue de 63 años (rango 45-83) y en el femenino de 68 años (rango 48-83), este último promedio superior estadísticamente $(\mathrm{p}<0,05)$, al de los hombres. La edad menor de los que cursaron con ISQ fue de 45 años, con 83 años en el extremo superior.

La proporción de mujeres operadas que se infectaron, en comparación con los varones en similares condiciones, fue también superior estadísticamente $(\mathrm{p}<0,05)$, por lo que, aun cuando se operaron menos, finalmente el sexo femenino se infectó más.

No se observó correlación entre ISQ y edad (Figura 1). El mayor número de ISQ se ubicó en 73 años y el $40 \%$ de las ISQ en los pacientes sobre 70 años.

En la evaluación del comportamiento de las variables individuales de ambas escalas, se observó que: clasificación de la herida operatoria, antibioprofilaxis, uso de arteria mamaria y circulación extracorpórea fueron similares y se distribuyeron proporcionalmente iguales y cercano al $100 \%$ en los casos y controles, por lo que se excluyeron de los análisis finales.

La escala Toronto clasificó en mediano/alto riesgo a $25 \%$ de los casos, proporción estadísticamente significativa en comparación a NNIS que incluyó en este riesgo sólo a 11\%, (p < 0,05, OR 2,94; IC 95\%: 1,47-5,90) (Tabla 2).

Al efectuar un modelo logístico con las constituyentes de estas escalas, se observó únicamente la variable "UCI mayor a 3 días" con peso 95\% de confianza en la asociación con ISQ, lo que no ocurrió con las variables de NNIS (Tabla 3). Se analizó por separado el comportamiento de estadía en UCI > 3 días y se identificó que esta se asoció además con edad $>64$ años $(\mathrm{p}<0,05)$.

Respecto a la capacidad de discriminación de la escala Toronto según sensibilidad y especificidad, a través de curva ROC, los pacientes clasificados en riesgo por Toronto presentaron una mayor probabilidad de cursar una ISQ que los que no clasificaron de igual forma (área bajo la

Tabla 1. Aporte de casos y controles de pacientes con cirugía bypass coronario por centro asistencial, Santiago 1998-2002

\begin{tabular}{cccc}
\hline Centro hospitalario & Casos $(\mathbf{n})$ & Controles $(\mathbf{n})$ & Participación (\%) \\
\hline 1 & 18 & 54 & 25,4 \\
2 & 12 & 36 & 16,9 \\
3 & 9 & 27 & 12,7 \\
4 & 15 & 45 & 21,1 \\
5 & 3 & 9 & 4,2 \\
6 & 14 & 42 & 19,7 \\
Total & 71 & 213 & 100 \\
\hline
\end{tabular}




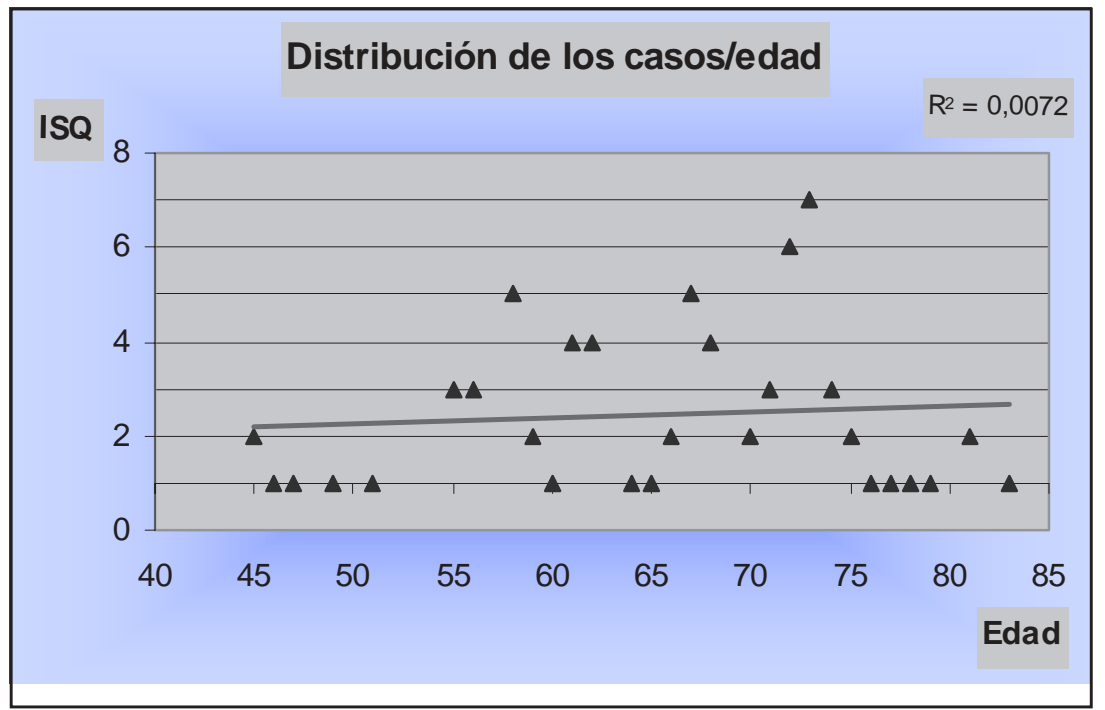

Figura 1. Correlación de las ISQ por edad en pacientes sometidos a bypass coronario, Santiago 1998-2002 ( $\mathrm{n}=71)$.

curva $=0,659)$. La misma evaluación gráfica para la escala NNIS reveló ausencia de discriminación (área bajo la curva $=0,511$ ).

La evaluación de los factores de riesgo propios del paciente mostró que la variable diabetes mellitus de la escala Toronto se asoció sólo con el sexo femenino $(p=0,04)$. En el análisis por $\chi^{2}$ se confirmó la asociación de ISQ con estadía en $\mathrm{UCI}>$ a 3 días y con clasificar en Toronto > 9 para ambos sexos (Tabla 4). No se encontró asociación de las ISQ con fallecimiento, por test exacto de Fisher.

Tabla 2. Análisis del comportamiento de las escalas NNIS y Toronto en pacientes sometidos a bypass coronario. Santiago 1998-2002 $(\mathrm{n}=71: 213)$

\begin{tabular}{ccccc}
\hline Escala & Casos identificados & OR & IC (95\%) & \multicolumn{1}{c}{ P } \\
\hline Toronto $>9,5$ & $18 / 71$ & 2,94 & $1,47-5,90$ & 0,002 \\
NNIS $>2$ & $8 / 71$ & 1,29 & $0,53-3,1$ & 0,56 \\
\hline
\end{tabular}

Tabla 3. Análisis por modelos logísticos de las subvariables por escalas NNIS y Toronto en pacientes sometidos a bypass coronario. Santiago 1998 - 2002

\begin{tabular}{ccccc}
\hline Escala $\backslash$ variable & OR & IC (95\%) & P & $\begin{array}{c}\text { Significación } \\
\text { estadística }\end{array}$ \\
\hline $\begin{array}{c}\text { Modelo Toronto: } \\
\text { Diabetes mellitus }\end{array}$ & 1,59 & $0,89-2,85$ & 0,11 & NS \\
$\quad$ Reintervención & 0,60 & $0,18-1,96$ & 0,40 & NS \\
UCI $>3$ días & 3,99 & $2,24-7,11$ & 0,00 & $\mathrm{~S}$ \\
Modelo NNIS: & & & & NS \\
T $>$ hs & 1,71 & $0,94-3,05$ & 0,07 & NS \\
ASA $>2$ & 1,13 & $0,65-1,97$ & 0,66 & \\
\hline
\end{tabular}

NS = No significativo $\mathrm{S}=$ Significativo $\mathrm{T}$ : tiempo operatorio ASA: índice de riesgo anestésico 
Tabla 4. Regresión logística de las variables de riesgo en ISQ de pacientes sometidos a cirugía de bypass coronario. Santiago 1998 - 2002*

\begin{tabular}{lcccc}
\hline Variable & OR & IC (95\%) & P & \\
\hline - Edad & 0,99 & $0,95-1,03$ & 0,72 & NS \\
- Sexo & 1,01 & $0,53-1,92$ & 0,96 & NS \\
- T $>5$ hs & 1,50 & $0,78-2,85$ & 0,21 & NS \\
- ASA $>2$ & 0,97 & $0,52-1,81$ & 0,94 & NS \\
- Diabetes mellitus & 1,54 & $0,85-2,78$ & 0,15 & NS \\
- Reintervención primeros 4 días & 0,57 & $0,17-1,94$ & 0,37 & $\mathrm{NS}$ \\
- UCI $>3$ días & 3,94 & $2,16-7,18$ & 0,00 & $\mathrm{~S}$ \\
- Bypass $>2$ & 1,56 & $0,82-2,96$ & 0,18 & $\mathrm{NS}$ \\
\hline
\end{tabular}

NS = No significativo $\quad \mathrm{S}=$ significativo $\quad \mathrm{T}=$ tiempo operatorio $\quad$ *Excluye Toronto y NNIS (Tabla 2)

\section{Discusión}

Este estudio constituye un primer intento por efectuar comparaciones ajustadas por medio de una previa evaluación de la aplicación de escalas puntuables predictoras de riesgo de ISQ en población chilena sometida a cirugía de bypass coronario. Esto ha permitido conocer algunos favorecedores del riesgo de infección nosocomial. Sin embargo, el incumplimiento del tamaño de muestra ideal calculado no permitió alcanzar un poder estadístico significativo. Tal situación fue secundaria al modelo retrospectivo en la obtención de la información y en que además se evidenció una falencia en los documentos y registros de las variables perseguidas. Durante la recolección de datos fue posible apreciar que la clasificación de la herida operatoria no es una práctica regular y que cuando se efectúa, varía incomprensiblemente según distintas definiciones de los equipos quirúrgicos o centros hospitalarios. Respecto al registro escrito del índice anestésico, ASA, es también irregular o en numerosos casos no concuerda con los criterios conocidos.

Resulta llamativo en este estudio el hecho estadísticamente significativo, que la infección sea más frecuente en mujeres, siendo que la cirugía cardíaca por cardiopatía coronaria es practicada mayoritaria en hombres.

Aún así, los resultados presentados apuntan a que el índice Toronto con puntaje mayor a 9, corresponde a la clasificación que mejor se asocia con el riesgo de ISQ en esternotomía en pacientes de ambos sexos operados de cardiocirugía de bypass en población chilena. De esta escala, permanencia en UCI por más de 3 días fue la variable que encontró la mejor correlación con el evento adverso nosocomial, lo que concuerda con los resultados obtenidos por la Unidad Cardiovascular del Hospital de Toronto.

Conocida ya esta variable del índice de riesgo Toronto, es altamente probable que en una muestra mayor en número se identifiquen también otras con carácter significativo como reintervención, que al tener una correlación significativa aportaría así a otro tipo de información paralela para la construcción de un indicador permanente de la calidad del evento operatorio, por ejemplo: número de bypass coronarios/número de reintervenciones asociadas, por 100 eventos

La escala NNIS cuenta, dado los resultados obtenidos en este estudio, con dos de las tres variables que se comportarían como sesgos de clasificación errónea no diferencial, factor importante a considerar por la alta probabilidad de subestimar la verdadera asociación existente entre las variables y las ISQ al ser aplicada como tal.

Respecto al índice Toronto, es probable que el tamaño de la muestra alcanzado haya impedido la identificación de componentes que no se comportaron como se esperaba. Esto se evidencia en la variable diabetes mellitus, donde el poder alcanzado fue de 55\% para los sexos agrupados, distribuyéndose esta variable con $61,5 \%$ en mujeres, en las que efectivamente encontró asociación.

En una población de estudio mayor en número, el poder individual aumentaría en cada una de ellas, pudiendo ser evidenciadas con certeza estadística del mismo modo que la asociación existente entre infección y fallecimiento, donde el tamaño de muestra aumenta sustancialmente so- 
bre lo calculado para este estudio. Se ha estimado en $\sim 14 \%$ la mortalidad asociada a los pacientes que desarrollan una mediastinitis ${ }^{14-16}$.

La aplicabilidad del índice Toronto es viable, cuanto más si se efectúa contemporáneamente al evento y evolución quirúrgica. La objetividad de sus componentes hace de este índice un evaluador concreto de la atención asistencial según las diferencias entre las tasas estratificadas de los centros asistenciales que efectúan este tipo particular de cirugía, reconociendo sin embargo, que aunque evalúa riesgo, no permite comparar poblaciones en su condición pre operatoria.

Es un aspecto a considerar, en una eventual futura puesta en práctica de la escala Toronto, el diferenciar diabetes mellitus de hiperglicemia perioperatoria, por cuanto ha sido esta última la frecuentemente señalada en asociación a las ISQ ${ }^{17}$.

Al no existir información nacional previa al respecto, este estudio representa un esfuerzo inicial por abrir la alternativa de una vigilancia estratificada en algunos procesos infecciosos nosocomiales de relevancia nacional por calidad, costos y accesibilidad como lo es en este caso, la cirugía de bypass coronario.

Agradecimientos: A los centros hospitalarios colaboradores, a sus directivos, profesionales, personal administrativo y otros, por haber permitido y facilitado la investigación. A los grupos conformados por los profesionales de los programas de Control de IIH y Epidemiología Hospitalaria, como también a todos aquellos que favorecieron y participaron altruistamente por el desarrollo y logro final. Para la publicación de este artículo un reconocimiento al Editor de la Sección Infecciones Intrahospitalarias de la Revista Chilena de Infectología, Luis Delpiano M, por las sugerencias y participación en la preparación del manuscrito.

\section{Resumen}

En un estudio piloto multicéntrico de caso-control (71/213) se investigó el comportamiento de dos índices predictores de riesgo de infección de sitio quirúrgico, NNIS y Toronto, en pacientes operados de bypass coronario en seis hospitales de Santiago de Chile, entre los años 1998 y 2002. Se identificó en la escala Toronto y en una de sus subvariables: estadía superior a tres días en Unidad de Cuidados Intensivos, una correlación positiva con el riesgo de infección de herida operatoria. La escala Toronto, con sus cuatro subclasificaciones de riesgos, es por lo tanto un efectivo índice predictor que permitiría al ajustar las tasas, efectuar comparaciones entre diferentes hospitales y centros que llevan a cabo este tipo de cirugía.

\section{Referencias}

1.- Araujo M. Listas de espera para cirugía cardíaca con circulación extracorpórea: Revisión de la literatura. Ministerio de Salud de Chile, Unidad Evaluación de Tecnología Sanitaria, 2002.

2.- Brenner P, Pohlenz M, Otaiza F. Informe de vigilancia epidemiológica de las infecciones intrahospitalarias, Chile 2002. División de planificación y presupuesto, Departamento de calidad de prestadores del Ministerio de Salud de Chile.

3.- Arcil G, Astorga J. Análisis y propuesta de desarrollo: Modelo de red cardiovascular nacional. Ministerio de Salud de Chile, 2003.

4.- Richards C, Emory T G, Edwards J, Fridkin S, Tolson J, Gaynes R. Characteristic of hospital and infection control professionals participating in the National Nosocomial Infections Surveillance System 1999. Am J Infect Control 2001; 29: 400-3.

5.- Farinas-Álvarez C, Farinas M C, Prieto D, DelgadoRodríguez M. Applicability of two surgical-site infection risk indices to risk of sepsis in surgical patients. Infect Control Hosp Epidemiol 2000; 21: 633-8.

6.- Russo P L, Spelman D W. A new surgical-site infection risk index using risk factors identified by multivariate analysis for patient undergoing coronary artery bypass graft surgery. Infect Control Hosp Epidemiol 2002; 23: 372-6.

7.- Kohli M, Yuan L, Escobar M, David T, Gillis G, Comm B et al. A risk index for sternal surgical wound infection after cardiovascular surgery. Infect Control Hosp Epidemiol 2003; 24: 17-25.

8.- Olsen M A, Lock-Buckley P, Hopkins D, Polish L B, Sundt T M, Fraser V J. The risk factors for deep and superficial chest surgical- site infections after coronary artery bypass graft surgery are different. J Thorac Cardiovasc Surg 2002; 124: 136-45.

9.- Gaynes R P. Surgical-site infections (SSI) and the NNIS Basic SSI Risk Index, part II: room for improvement. Infect Control Hosp Epidemiol 2001; 22: 266-7.

10.- Culver D H, Horan T C, Gaynes R P, Martone W J, Jarvis W R, Emori T G, et al. Surgical wound infection rates by wound class, operative procedure, and patient risk index. National Nosocomial Infections Surveillance System. Am J Med 1991; 91 (3B): 152-7S.

11.- Gaynes R P. Surgical-site infections and the NNIS SSI Risk Index: room for improvement. Infect Control Hosp Epidemiol 2000; 21: 184-5.

12.- Dripps R D, Eckenhoff J E, Vandam L D. Anestesiología. 7th ed. 1998. Editorial Interamericana, sección 2, capítulo 2, 1988; pp: 15.

13.- Del Valle I, Dosta J, Robles A. Variación de criterios entre los anestesiólogos sobre la clasificación del estado físico según la Sociedad Americana de Anestesiología. Rev Anest Mex 1999; 11: 150-5.

14.- Softah A, Bedard A, Hendry P, Goldstein W, Brais M, Keon W. Wound infection in cardiac surgery. Ann Saudi Med 2002; 22: 105-7.

15.- Clara L, Stern L, Barcan L, Marchetti M, Greco G. 
Mediastinitis post-quirúrgica: Estudio caso-control. Rev Chil Infect 2002; 19: 37-48.

16.- Wanke H, Kruse J, Mohn W. Nosocomial infections and sternum osteomyelitis. Zentralblatt fur Chirugie 1990; 115: 563-8.
17.- Estrada C A, Young J A, Nifong L W, Chitwood W R Jr. Outcomes and perioperative hyperglicemia in patients with or without diabetes mellitus undergoing coronary artery bypass grafting. Ann Thorac Surg 2003; 75: 1392-9.

Correspondencia a:

Patricia López Flores

iihplf@hosmil.cl

patricialopez@vtr.net 